\title{
Optimization Model and Method of Variable Speed Limit for Urban Expressway
}

\author{
Shubin Li $\mathbb{D}$, ${ }^{1}$ Tao Wang $\mathbb{D}^{2,}$, Hualing Ren $\mathbb{D}^{3},{ }^{3}$ Baiying Shi $\mathbb{D},{ }^{4}$ and Xiangke Kong $\mathbb{C}^{4}$ \\ ${ }^{1}$ Department of Traffic Management Engineering, Shandong Police College, Jinan 250014, China \\ ${ }^{2}$ Department of Automation and Electronic Engineering, Qingdao University of Science and Technology, Qingdao 266061, China \\ ${ }^{3}$ MOE Key Laboratory for Urban Transportation Complex Systems Theory and Technology, Beijing Jiaotong University, \\ Beijing 100044, China \\ ${ }^{4}$ Department of Traffic Engineering, Shandong Jianzhu University, Jinan 250101, China
}

Correspondence should be addressed to Shubin Li; li_shu_bin@163.com, Tao Wang; wangtao@qust.edu.cn, and Baiying Shi; shibaiying@sdjzu.edu.cn

Received 26 March 2021; Revised 20 April 2021; Accepted 5 May 2021; Published 19 May 2021

Academic Editor: Yong Wang

Copyright (c) 2021 Shubin Li et al. This is an open access article distributed under the Creative Commons Attribution License, which permits unrestricted use, distribution, and reproduction in any medium, provided the original work is properly cited.

The urban expressway network is the main part of the urban traffic network carrying most of the city's traffic pressure for its continuity and rapidity, but the control method of the traffic flow was too simple to other control methods in application in addition to the ramp control and the fixed speed control. In this paper, the theory of variable speed limit (VSL) was used to develop an optimal control model based on the improved traffic flow simulation model according to the characteristics of urban expressway traffic flow. The objective of the proposed model is to minimize the delay and maximize the traffic flow. It can adjust the traffic flow on the network in space time so that the whole network is in a state of equilibrium which not only is conducive to the control of the local traffic congestion and avoids the spread of congestion but also improves the traffic safety. The SPSA-based solution algorithm was proposed by taking into account the needs of real-time online applications. It can not only ensure the accuracy of the solution but also meet the requirements of the simulation time. The simulation results show that the variable speed limit can be optimized in moderate demand, and the proposed model and algorithm are effective and feasible in this paper. The conclusions are useful to help the traffic management department to formulate reasonable traffic control strategies.

\section{Introduction}

Thanks to the rapid development of economy and mechanization, traffic congestion has become one of the most prominent city problems in China. It gradually spreads from big cities to medium-sized and small ones. Even metropolises like Beijing, Shanghai, Guangzhou, and Shenzhen have not conquered the traffic congestion and therefore they are nicknamed "congestion city." The Annual Report of Beijing Traffic Development of 2015 shows that the traffic congestion becomes worse per hour, and the traffic flow of the central part of Beijing increased by $2.7 \%$ compared to that of the previous year. The time quantum of congestion is one hour and fifty-five minutes and the distance of severe traffic congestion is $10.4 \%$. Apparently, this worldwide problem has caused Chinese top leaders concern. President Xi Jinping listened to relevant reports while he was inspecting the traffic in Beijing. He demanded that the transport sector analyzes the traffic problems and performs effective measures, which promotes the efforts and research to relieve the noncapital function of Beijing and the scheme of coordinated development of the Beijing-Tianjin-Hebei region. Developing the intelligent transportation system is one of the strategies to approach traffic congestion, namely, balancing the conflict between the traffic demand and the traffic supply by virtue of modernized science and technology, together with macroregulation of government. In this way, it is likely to achieve smooth transportation. Taking the traffic of Beijing as a case in point, each car serves 1.99 people daily before the appearance of an intelligent travel 
platform which can serve 2.24 people each day. The availability of cars increases by $12.1 \%$. In October 2014, Chinese Premier Li Keqiang pointed out that the so-called intelligent sustainable transportation should be given a complete definition. It refers to addressing the traffic problems through the combination of traffic factors (people, car, and road) and the achievement of intelligent traffic through education, law enforcement, and technology. Recently, Huabei Area Intelligent Travel Big Data Report released by Uber indicates that the first five months of 2014 have witnessed 3.5 billion people in Huabei province who used the intelligent travel application when going out. Beijing, Taiyuan, and Tianjin take the lead in the employment of this app among cities in Huabei area. The focal point in the study of intelligent traffic technology lies in the evaluation and prediction of the current traffic network and the implementation of online control.

As the main part of the urban traffic network, the urban expressway bears the heaviest urban traffic pressure and is characterized by high speed, large flow, and continuous traffic flow. However, more effective methods are in urgent demand for the management of urban expressway because it is not compatible with those for the management of highway and for the tackling of conventional traffic problems. Hence, it is a significant try to introduce the intelligent control of variable speed limit (VSL) to the management of urban expressway.

The management of variable speed limit is subject to current traffic conditions, which requires sense and judges the condition of the traffic network by checking the current and previous data. The major concern about the evaluation of the traffic network lies in the use of the traffic flow simulation model which can be roughly divided into macrotraffic flow simulation model [1], mesotraffic flow simulation model [2, 3], microtraffic flow simulation model [4], and all kinds of the optimized simulation model [5-8]. Among them, the mesotraffic flow model can simulate different levels of traffic details with its unique modeling ideas and simulation methods. Considering the computational accuracy and speed, the mesotraffic flow model is of great significance in the study of the intelligent transportation system (ITS) and hybrid simulation model. Felipe proposed an agent-based mesoscopic traffic flow simulation model, which could satisfy both the macrosimulation efficiency and the microsimulation accuracy [9]. Anahita proposed a midrange flow-emission model considering the aggregation behavior of different vehicle groups, which verified that the model was suitable for real-time simulation [10].

Making positive traffic control is based on the online simulation of the real-time traffic condition through simulated dynamic characteristics. Currently, the function of maintaining traffic safety is analyzed in most studies of variable speed limit [11-14]. As a part of positive traffic management, the variable speed limit can optimize and control the speed of the traffic flow in the artery movement, thus maintaining traffic safety and order. However, little emphasis is put on the study of alleviating traffic congestion. Zhang established a cellular automaton model in highway variable speed limit zone to study the effect of speed limit value and speed limit zone on traffic congestion [15]. Wang $\mathrm{W}$ regarded the variable speed limit control of expressway as the discrete-time Markov decision process, proposed the variable speed limit control model based on reinforcement learning and Markov decision, and used the Paramics simulation software to verify that the model can improve the traffic flow [16]. Zhou proposes a variable speed limit control strategy based on reinforcement learning and verifies that the strategy has obvious advantages in reducing congestion and travel time [17].

In order to relieve the traffic pressure and realize the change from passive reactive traffic management to active precautionary traffic management, this paper focuses on how to achieve traffic safety and smooth traffic flow. The main innovations of this paper include the following: (1) In view of the traffic jam of Jinan viaduct expressway, an improved medium traffic flow model more suitable for local traffic conditions is adopted to simulate the movement of vehicles. (2) In order to achieve the effect of system optimization, an optimization model of variable speed limit is proposed to adjust the speed, so as to achieve the balanced distribution of traffic density and promote the improvement of traffic efficiency. (3) e solution algorithm based on SPSA is designed to help the traffic management department formulate reasonable traffic control strategies.

\section{The Improved Mesoscopic Traffic Flow Simulation Model}

The mesoscopic traffic flow simulation model quickly arouses scholars' interest in traffic flow field because it can not only ensure the accuracy of simulation but also satisfy the demand for calculating speed of real-time online simulation. The anisotropic mesoscopic traffic flow simulation model in literature [3] is adopted in this paper and the model is improved according to speed limit control strategy. The construction of the mesoscopic traffic emulator usually includes four categories of models: the road network model, the static queuing model, the speed model, and the vehicle movement model.

2.1. The Road Network Model. The analysis method based on the network model is widely used in the fields of social, transportation, and logistics, such as logistics distribution network [18] and state space-time network [19, 20]. By constructing the network topology of the system, the evolution rule and essence of the system can often be reflected. The urban expressway network is composed of ramp and artery. Owing to the similarity with the freeway, the node, the connecting link, the lane group, the lane, detector, the turning prohibition line, and the traffic zone can be used to describe the topological structure of the urban expressway. As Figure 1, a road consists of the following elements: node, connecting line, lane, lane group, and segment. And traffic network consists of node and link. 


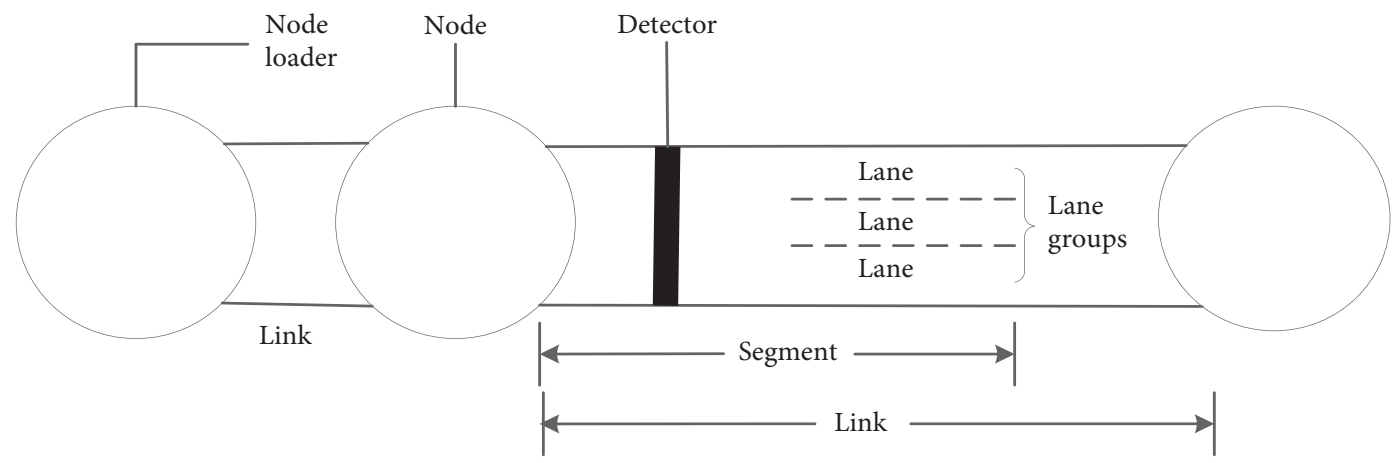

FIGURE 1: Road network topology.

The mentioned factors have a relationship as in Figure 2. VMS is the variable message signs, while VSL is the variable speed limit system.

2.2. The Static Queuing Model. When the expressway exit ramp or bottleneck section is congested, the application of this model to the upstream section of the congested section can effectively alleviate the traffic congestion. The vehicle behavior is similar to the queue in signal control intersection. It is necessary to calculate the process when a vehicle joins a queue, the movement in the queue, and the process when the vehicle leaves the queue. Suppose a vehicle $i$ is in a queue; then the queue will definitely incur delay which can be signified by the following formula:

$$
T=\frac{i}{c}
$$

According to what is mentioned in Figure 2, to define sections of a road with identical physical property as a segment, the lane group can be defined on the basis of segment. Generally, a lane group consists of one or more lanes. Then, $c$ represents the output capacity. On this segment, the vehicles are divided into moving ones and queuing ones and the above model is used to describe queuing behavior. $t$ refers to time, and then $c t$ refers to the number of vehicles that leave the lane group. Suppose a vehicle reaches the end of a queue at $t$; then its position can be expressed by the following formula:

$$
q(t)=q(0)+l_{\mathrm{car}}(c t-m)
$$

where $q(0)$ is the end position of queue at $t=(0) . l_{\text {car }}=$ $\left(1 / k_{\text {jam }}\right)$ represents the standard vehicle length including headway. $k_{\text {jam }}$ refers to the jam density. $m$ refers to the total number of vehicles between the one at the end of the queue and the considered one. That is to say, it refers to the number of vehicles that join the queue before the considered one.
It is worthy of note that $L$ refers to the length of segment and the model is worked with $0<q(t)<L$. When $q(t) \geq L$, the queue dissipated. This means that the considered vehicle failed to catch up with the queue and it is still in movement. Because there cannot be a negative number, $q(t)<0$ never occurs.

2.3. The Speed Model. Vehicles move at a certain speed on segment. The speed model can describe a vehicle with a certain speed in the network. According to the definition of the anisotropy mesoscopic traffic flow, the speed of the vehicle is determined by the traffic density of some areas downstream. In other words, the vehicles in movement are influenced by the ones in front of them. This influence fades with the increase of distance and has nothing to do with the vehicles left behind. Therefore, the anisotropy in mesoscopic traffic flow can be achieved through the definition of the length of the downstream region, which is called Speed Influencing Region (SIR). It is shown in Figure 3.

The traffic density of each vehicle in movement can be calculated according to the amount of vehicle in SIR. The first case is shown in Figure 3(a). The density of vehicle $i$ in SIR is as follows:

$$
k_{i}^{t-1}=\frac{N_{i}^{t-1}}{n l},
$$

where $N$ refers to the amount of vehicle $i$ in SIR when the time is $t-1, n$ refers to the amount of lane in SIR, and $l$ refers to the length of SIR. From 3(b) and 3(c), the formula of the region density is as follows:

$$
k_{i}^{t-1}=\min \left[k_{\mathrm{jam}} \frac{N_{i}^{t-1}}{m x+n(1-x)}\right] .
$$

According to the density of SIR, the current speed of vehicle $i$ can be calculated by means of the following exponential function:

$$
V_{i}^{t}= \begin{cases}V_{f}^{i}, & k \leq k_{\max }, \\ \max \left\{V_{\min }, V_{\max }\left\{1-\left(\frac{V_{i}^{t-1}-k_{\max }}{k_{\text {jam }}}\right)^{\beta}\right\},\right. & k>k_{\max },\end{cases}
$$




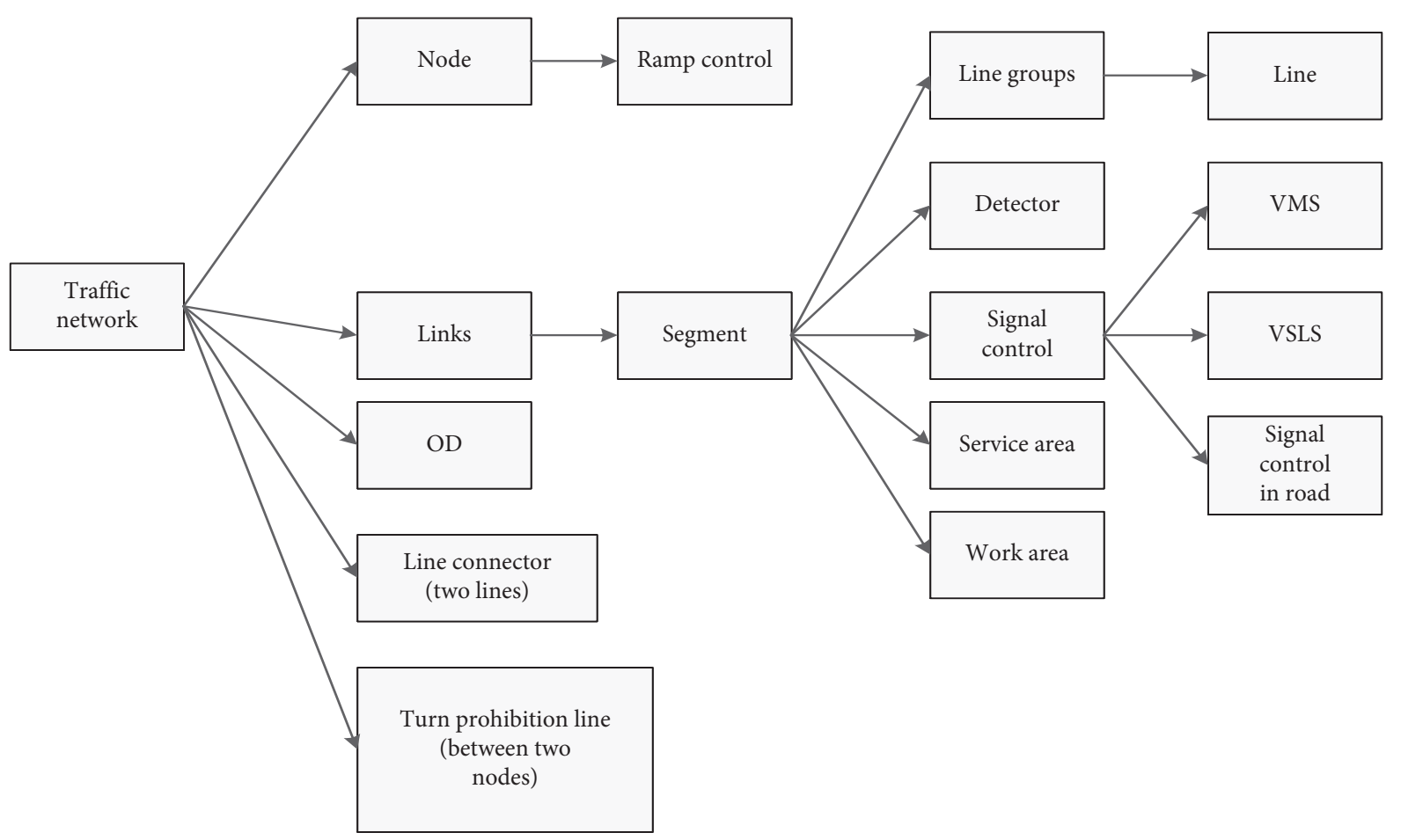

Figure 2: The relationship between network elements.

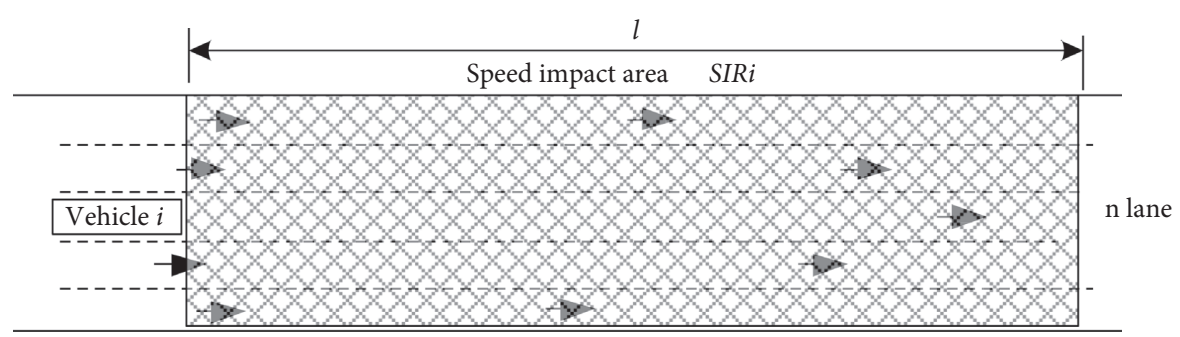

(a)

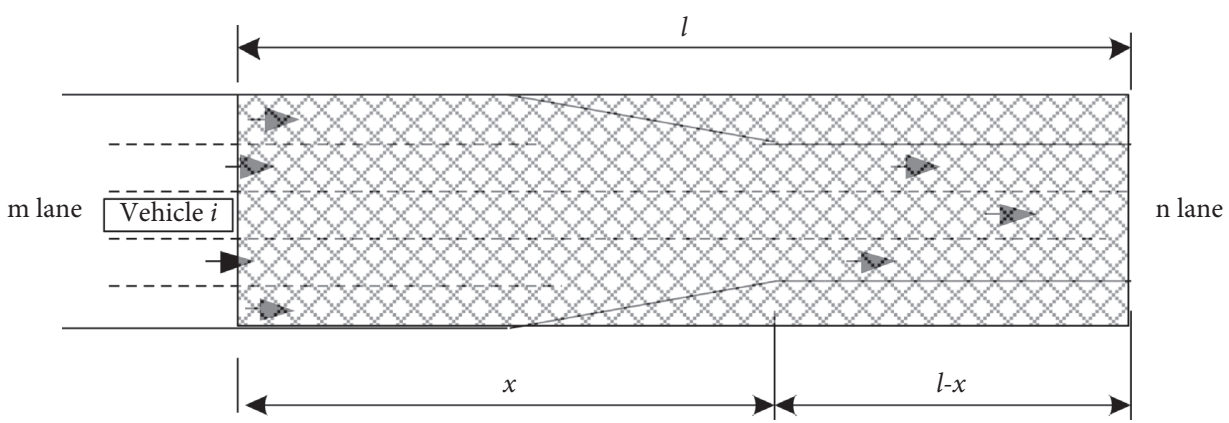

(b)

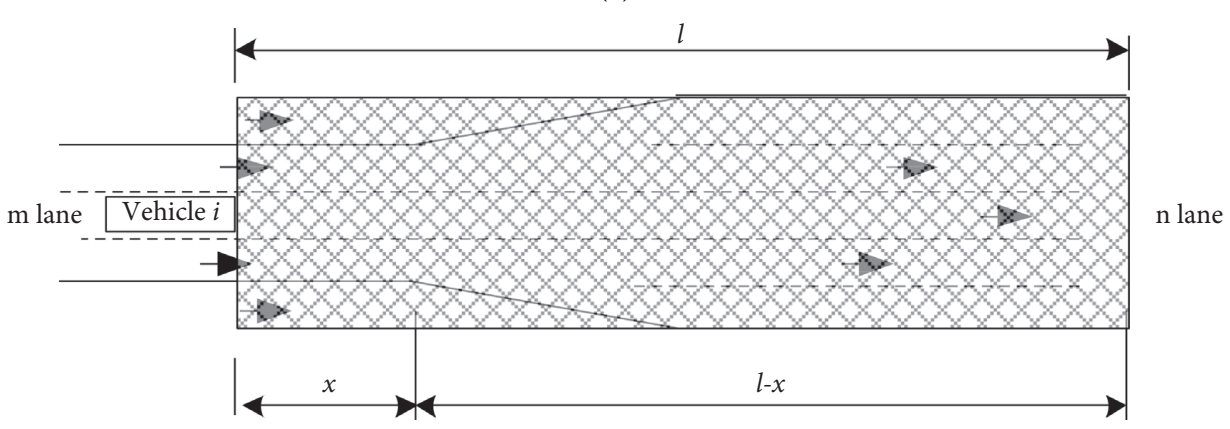

(c)

FIGURE 3: SIR of the Model. (a) Equal number of lane road sections. (b) Reduced number of lanes roads. (c) Increased number of lanes roads. 
where $V_{i}^{t}$ refers to the speed of vehicle $i$ at the time of $t ; V_{f}^{i}$ refers to the free flow speed on this segment; $V_{\min }$ refers to the minimum speed that can enable free flow to move; $\alpha$ and $\beta$ refer to model parameter. The relation between speed and density is shown in the following Figure 4.
The above formula demonstrates the state of the traffic flow without any control. Once there is a VSL control, the vehicles have to obey the speed limit. The model about the relation between speed and density changes.

$$
v_{i}^{t}= \begin{cases}\min \left(v_{f}^{i}, v_{\mathrm{lim}}^{i}\right), & k \leq k_{\max }, \\ \max \left\{v_{\min }, \min \left(v_{\max }, v_{\mathrm{lim}}^{i}\right)\left\{1-\left(\frac{\min \left(v_{i}^{t-1}, v_{\mathrm{lim}}^{i}\right)-k_{\max }}{k_{\mathrm{jam}}}\right)^{\beta}\right\},\right. & k>k_{\max } .\end{cases}
$$

2.4. The Vehicle Movement Model. The speed of vehicle is determined by the density of SIR in front of him when it moves on the segment. If there is no queuing, the vehicle is positioned at $z_{0}$ when $t=0$. Then the vehicle will arrive at $z$ when the time is $t(z)$. Their relation is shown as follows:

$$
t(z)= \begin{cases}\frac{1}{\lambda} \log \frac{\lambda_{z+v_{u}}}{\lambda_{z_{0}+v_{u}}}, & \text { if } v_{u} \neq v_{d} \\ \frac{z-z_{0}}{V_{u}}, & \text { if } v_{u}=v_{d}\end{cases}
$$

where $\lambda$ is determined by the following formula, $\lambda=\left(v_{d}-v_{u}\right) / L_{s} ; V_{d}$ refers to the downstream speed of the segment; $V_{u}$ refers to the upstream speed of the segment; $L_{s}$ refers to the length of segment $s$. Suppose the time $t=0$, the vehicle is positioned at $z_{0}$, then the position of vehicle is signified by the following formula when the time is $t$ :

$$
z(t)= \begin{cases}e^{\lambda t}\left(z_{0}+\frac{v_{u}}{\lambda}\right), & \text { if } v_{u} \neq v_{d}, \\ v_{u} t+z_{0}, & \text { if } v_{u}=v_{d} .\end{cases}
$$

If there is a queue on segment when the vehicle is positioned at $z(t)$, then the position of the vehicle is signified by the following formula when $t$ refers to any time:

$$
\begin{aligned}
& z(t)=e^{\lambda(t) t}\left(z_{0}+\frac{V_{u}}{\lambda(t)}\right)-\frac{V_{u}}{\lambda(t)}, \\
& \lambda(t)=\frac{-V_{u}}{q_{0}+l(c t-m)} .
\end{aligned}
$$

The meaning of $q_{0}, l, c, m$ is identical with that of the above formula.

If there is a VSL, the above formula is signified as follows:

$$
\begin{aligned}
& z(t)= \begin{cases}e^{\lambda(t) t}\left(z_{0}+\frac{\min \left(V_{u}, V_{\lim }\right)}{\lambda(t)}\right)-\frac{\min \left(V_{u}, V_{\lim }\right)}{\lambda(t)}, & \text { if } t<t^{*} \\
q_{0}+l(c t-m) & \text { if } t>t^{*},\end{cases} \\
& z(t)=e^{\lambda(t) t}\left[z_{0}+\frac{\min \left(V_{u} V_{\lim }\right)}{\lambda(t)}\right]-\frac{\min \left(V_{u}, V_{\lim }\right)}{\lambda(t)}, \\
& \lambda(t)=\frac{-\min \left(V_{u}, V_{\text {lim }}\right)}{q(0)+l(c t-m)} .
\end{aligned}
$$

\section{The Improved VSL Simulation Model}

3.1. The Optimization Model. Every connected link of the urban expressway network is defined as several segments. The segment set defined as $I, i$ is one of them. The simulation time $K$ is divided into equally several intervals, and each interval is signified by $k$. Apparently, $i \in I, k \in K$. Each segment has installed a monitor which includes a variable speed limit sign to obtain the speed, density, flow, and occupancy. The speed, density, and flow of the segment $i$ in the time interval $k$ are signified by $V_{i}(k), \rho_{i}(k)$, and $f_{i}(k)$, respectively. $\vartheta_{i}(k)$ means occupancy; the direction where $i$ decreases is the vehicle moving one. The speed limit of the time interval $k$ in segment $i$ is $V_{\text {lim }}(i, k)$. The target function studies the largest traffic flow of the whole network during the time interval. Then the improved variable speed limit simulation model is signified by the following formula: 


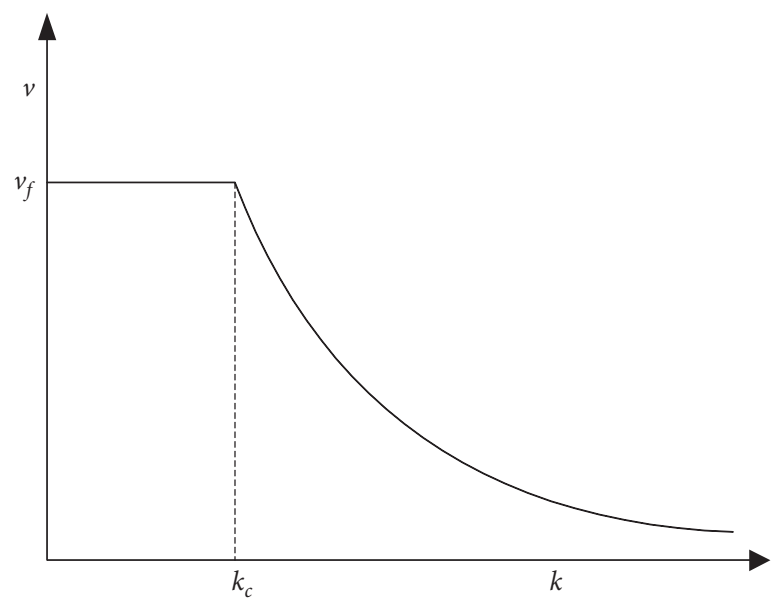

FIgURE 4: Speed-density curve in SIR.

$$
\begin{aligned}
\max & \sum_{k} \sum_{i} f_{i}(k), \\
V_{\lim }(i, k) & =\{20,30,40,50,60,70,80,90\}, \\
V_{\lim }(i, k) & \leq V_{\text {uplim }}(i, k), \\
i & =1,2, \ldots N ; k=1,2, \ldots K .
\end{aligned}
$$

$V_{\text {uplim }}(i, k)$ refers to the maximal value of the speed limit calculated according to the traffic of simulation time interval.

$$
\begin{aligned}
\vartheta(k) & =f\left(\vartheta_{i}(k, l), \vartheta_{i}(k, 2), \ldots, \vartheta_{i}\left(k, m_{i}\right)\right), \quad i=1,2, \ldots, N ; k=1,2, \ldots K, \\
V_{i}(k) & =f\left(\rho_{i}(k)\right), \quad i=1,2, \ldots, N ; k=1,2, \ldots K .
\end{aligned}
$$

$m_{i}$ refers to the amount of the detector group included in segment $i$

$$
\begin{aligned}
\rho_{i}(k) & =f\left(\vartheta_{i}(k)\right), \quad i=1,2, \ldots, N ; k=1,2, \ldots K, \\
V_{\lim }(i, k)-V_{\lim }(i-1, k) & \leq p(i), \quad i=1,2, \ldots, N ; k=1,2, \ldots K .
\end{aligned}
$$

$p(i)$ refers to the speed difference between adjacent sections of expressway. Hence, the VSL value is determined by the following formula:

$$
V_{\text {lim }}(i, k)=\min \left\{V_{\text {uplim }}(i, k), V_{\text {lim }}(i-1, k)+p(i), V_{i}(k)\right\} \text {. }
$$

3.2. The SPSA-Based Solution Algorithm. The conventional analytic algorithm can be used to solve the proposed model, such as the Newton algorithm and the projection algorithm. However, for real-time application, the mesoscopic traffic emulator is adopted to achieve online optimization of VSL control, so it is necessary to meet the real-time requirement of simulation as well as the accuracy of the calculation. Fortunately, the Simultaneous Perturbation Stochastic Approximation algorithm (SPSA) algorithm can cover all the characteristics listed above and it becomes the first choice for the real-time online simulation. It disturbs one time the vector components to be optimized at the same time and has nothing to do with the number of optimized parameters. Compared with the finite-difference stochastic approximation algorithm, its computing capability improves dramatically, which plays an essential role in optimization of largescale and numerous parameters. The SPSA should reevaluate the gradient vector in each iterative process while the algorithm only needs to perform calculation of objective function twice, which has nothing to do with the amount of parameter $n$ that needs estimating. This feature implies that the algorithm is applied to large-scale optimization.

The SPSA produces a series of estimated parameter values that make the gradient of objective function tend to be 0 . Then the updated formula of iteration for the $i$ time is as follows: 


$$
\theta^{i+1}=\theta^{i}-a_{i} \widehat{g}\left(\theta^{i}\right)
$$

where $\theta^{i}$ refers to the parameter vector when the algorithm starts to iterate for the $i$ time; $\hat{g}\left(\theta^{i}\right)$ refers to the estimated value of gradient vector; $a_{i}$ refers to the step length. The approximation of gradient can be obtained through two function evaluations by SPSA. The formula for that is as follows:

$$
\hat{g}\left(\theta^{i}\right)=\frac{z\left(\theta^{i}+c^{i} \Delta_{i}\right)}{2 c^{i}}\left[\begin{array}{c}
\Delta_{i 1}^{-1} \\
\Delta_{i 2}^{-1} \\
\vdots \\
\Delta i k
\end{array}\right] .
$$

The most striking advantage of the above formula lies in the fact that the calculational value of each iterative step is identical, which indicates that it has nothing to do with latitude $k$. The reason is that $\Delta i$ is the random perturbation of latitude $k$, and its numerator keeps identical to $k$ no matter if it is $1,2,3$, or more. Of course, if an algorithm wants to have a better application value, then it needs a reasonable number of iterations to achieve convergence. The detailed steps of the SPSA algorithm designed in this paper are as follows:

(i) Step 1. Initialization: set $i=0, \theta^{i}=\theta^{0}$ is the initial value. In actual practice, set a list of nonnegative parameters $[21,22]$ as the variable speed limit value. At the same time, initialize network state, define traffic network, node, and division of segment, and load on traffic demand and the initiative value of variable speed limit.

(ii) Step 2. Set the times of grad-rep for the calculation of gradient vector in each iteration; namely, choose the average value as the gradient estimated value in each step of iteration.

(iii) Step 3. Let $i=i+1$, and calculate formula (16) again.

(iv) Step 4. Produce $\Delta_{j}$, the disturbance random vector of latitude $k$. Each element $\Delta_{j k}, k=1,2, \ldots, k$, is obtained through random selection of Bernoulli distribution. The density function of this probability distribution is symmetrical about the vertical axis. And there is an upper bound for $\left|\Delta_{j k}\right|$ and $E\left|\Delta_{j k}^{-1}\right|$. This upper bound is in accordance with the characteristic of variable speed limit studied in this paper.

(v) Step 5. Evaluate the target function of variable speed limit model by the mesoscopic traffic model proposed in this paper; obtain value from $\theta^{i+}=$ $\theta^{i}+c^{i} \Delta_{j}$ and $\theta^{i-}=\theta^{i}+c^{i} \Delta_{j}$. Each point can satisfy the upper and lower bound of optimization. The following mesoscopic traffic simulation is as follows:

(a) Step 5.1. Define the update time interval and advance time interval. The relation is as follows:

$$
\begin{aligned}
T & =k_{u} \Delta t_{\text {update }}, \\
\Delta t_{\text {update }} & =k_{A} \Delta_{\text {advance }} .
\end{aligned}
$$

(b) $k_{A}$ and $k_{u}$ are positive integer, and $T$ is the simulation time. The specific parameter of the traffic flow model is mainly updated during update time and the vehicles are moved to a new place in advance interval.

(c) Step 5.2. Simulation circulation starts, and the update time interval is utilized. Counter $c$ plus $1, c=k_{u}$, happens to discontinue the algorithm.

(d) Step 5.3. Optimization circulation starts to output the information about the traffic state, such as the average flow, speed and density of the segments, and the length of the queue. Return to step 5.2.

(vi) Step 6. Calculate the gradient vector of latitude $k$ random approximation according to formula (15). The identical numerator of $k$ component serves as the essential content that distinguishes it from finite difference.

(vii) Step 7. Repeat step 4 to step 6 for grad-rep times, and each time draw sample independently for $\Delta_{j}$. Finally, calculate the average value at $\theta^{i}$.

(viii) Step 8. Obtain a new solution point at $\theta^{i+1}$. Then make adjustment according to the question.

(ix) Step 9. Return to step 3, and iterate to convergence. The condition for convergence is that the iterative times reach the upper bound, or the function value corresponding to $\theta^{i}$ keeps stable in several continuous iterative processes. Then iteration stops.

Literature [21] indicates that the most satisfactory convergence speed of the SPSA algorithm is around $k^{1 / 3}$. As direction of search is randomly chosen, hence it cannot be ensured that every step of iteration is downward. But it can be ensured that expectation is unbiased estimate:

$$
E\left[\hat{g}\left(\hat{\theta}_{k}\right)\right]=g\left(\hat{\theta}_{k}\right)+b^{k} \text {. }
$$

$b^{k}=u c^{k^{2}}, u$ is a constant. As opposed to the comparatively large $k$, the deviation $b^{k}$ will gradually disappear with $c^{k}$ approaching 0 . Therefore, it can be concluded that the outcome is closed to the optimal one.

\section{Results and Discussion}

4.1. Subheadings. The traffic network composed of three elevated expressways of Jinan in Shandong province is adopted as a case study. The three elevated expressways are Beiyuan elevated expressway, Shunhe elevated expressway, and east Erhuan elevated expressway, respectively. Especially, Beiyuan elevated expressway is $13.6 \mathrm{~km}$, including 8 pairs of ramp entrances and exits and 6 two-way lanes; east Erhuan elevated expressway is $9.5 \mathrm{~km}$ including 4 entrances and exits and 4 two-way lanes; Shunhe elevated expressway is $7.4 \mathrm{~km}$ and its width is $18.5 \mathrm{~m}$, including 4 lanes. As one of 
Jinan first-class arteries, its total area is $\mathbf{1 2 . 2 5}$ million square meters, including 1 toll station, 7 entrances and exits, and 3 interchange overpasses. The simplified diagram of traffic network is shown in Figure 5.

In this network, the diamond marked with number represents node, and it also represents point OD for the traffic demand, namely, the place where vehicles appear and disappear. For the convenience of simulation, we define the lanes between nodes which have unified physical characteristics; namely, they can be defined as a segment. The focus of this paper is how the VSL control method diffuses congested vehicles and seeks the law of traffic flow diffusion. In order to simulate the real traffic situation and reveal its essence, the parameters of the traffic flow model are set according to the specific characteristics of the road section (length, width, level, capacity, and traffic flow), where the free flow speed is $90 \mathrm{~km} / \mathrm{h}$, the defined $\rho_{\text {jam }}$ is 0.1150 , and $\alpha$ is 3.500 , and $\beta$ is 0.75004 . The capacity for input and output of each segment is 0.6667 per second per car. The time for step length predicted and estimated by the dynamic OD is 15 minutes. The whole simulation lasts for $3 \mathrm{~h}$. This means that there are 12 simulation time intervals. All areas of SIR influenced by heterogeneous directions of the mesoscopic traffic flow simulation model are defined as $150 \mathrm{~m}$. The time for advance step length is $10 \mathrm{~s}$ and for update step length is $20 \mathrm{~s}$.

It deserves to explain that emphasis in this paper is exerted on the test of effect and feasibility of the proposed VSL control strategy. Hence set the ideal demand level of dynamic OD, and adopt a periodic loading method that is characterized by a closed cycle. At the beginning of each simulation cycle, the dynamic OD demand is loaded on the network. The normal distribution is adopted to load vehicles to the corresponding point in the network when vehicles are loaded to node or segment in loading point. The VSL cannot perform a function on traffic flow with high density or low speed, nor can it exert influence on fluent traffic network. Thus it only works for moderate demand. Due to the shortage of the real OD demand data, different demand levels are tested to figure out the amount of OD adopted in the simulation. When all demands are set as 1 per interval, all the connected lines in the network appear green, which shows that the traffic runs smoothly. Then the demand is in order turned to $2,3,4$, and 5 , and the results show that the traffic network is relatively smooth, smooth, slight congestion, and congestion. Therefore, the demand of 4 is selected in this paper as the demand level studied concerning variable speed limit control. Additionally, 30\% random disturbance is complemented in each time interval of the traffic demand. That is to say, there is $30 \%$ random change of time demand by OD in each time interval.

During simulation, all the segments of expressway network are installed with detection, on which the average density, speed, and flow of the traffic flow can be perceived and computed. The variable speed limit value can be shown on VMS installed on roadside with comparatively long physical length to limit the speed of vehicles. Also, it is wise to select the integer among all the variable speed limit values displayed on the VMS; for example, the computed speed of the optimized model is $37 \mathrm{~km} / \mathrm{h}$. In this case, displaying $40 \mathrm{~km} / \mathrm{h}$ is a better choice. In terms of the network of Jinan elevated expressway, it is highly admirable to set $6 \mathrm{VMS}$, to limit speed from 40 to $60 \mathrm{~km} / \mathrm{h}$, and to select integral 10 digits. The simulation process is conducted by one laptop $4 \mathrm{G}$ internal storage. First, execute non-variable speed limit network. Then execute the variable speed limit strategy process. Finally, execute the network of optimal variable speed limit strategy. It is shown in Figure 6.

There are two aspects of practical significance in this study. On the one hand, the variable speed limit control proposed in this paper can achieve the purpose of stabilizing traffic flow. According to the traffic condition of the elevated road network, the dynamic adjustment of the speed limit value can achieve the purpose of balanced distribution of traffic flow and improve traffic safety. On the other hand, according to the traffic condition of the urban main road, the traffic efficiency of the urban main road can be improved by adjusting the traffic flow near the ramp exit of the viaduct through variable speed restriction.

4.2. The Simulation Results. Firstly, build the standard state of the traffic network without any control. Then observe its macro- and microcharacteristics. Secondly, set control strategy of VSL and observe the macro- and microcharacteristics of the traffic network with the same demand. Lastly, operate the optimal model of the VSL and the algorithm, also observe and discuss the macro- and microcharacteristics of the traffic network after optimization. From the macroscopic view, the major concern is carried on the observation of the total flow, the total density, and the total speed on the network during the simulation time. Observation of the distribution and variety of the average density, the average speed, and the average flow on each road during each simulation time interval become the priority from the microscopic aspect. At the same time, the traffic impedance of the three cases is compared.

As previously mentioned, the traffic demand in each time interval is defined as 4 . Then perform $30 \%$ random disturbance to build the standard state of the traffic network. In this way, the traffic demand in each interval is subtlety different. Under this standard, the time of the whole simulation witnesses the following aspects: the total travelling time is $2759.7 \mathrm{~h}$, the total travelling distance is $156169.3 \mathrm{~km}$, the total average speed is $42.6 \mathrm{~km} / \mathrm{h}$, the total traffic flow is 188399 vehicles, the total delay is $879.1 \mathrm{~h}$, the largest delay on node is $153.4 \mathrm{~h}$, the total density is 4.5412 , and the average flow is 96.0930. In terms of the microscopic aspect, the distribution of the average density, the average speed, and the average flow in each segment is shown in Figures 7-9.

Under the above standard state, the initial VSL control scheme is set. The Beiyuan elevated expressway, the Shunhe elevated expressway, and the Erhuan elevated expressway are installed with eight VMS, respectively, including four in each direction. These VMS are placed on the middle part of the road with comparatively long physical length with the aim of controlling the speed of the traffic flow. Considering the traffic safety and the traffic congestion of the elevated 


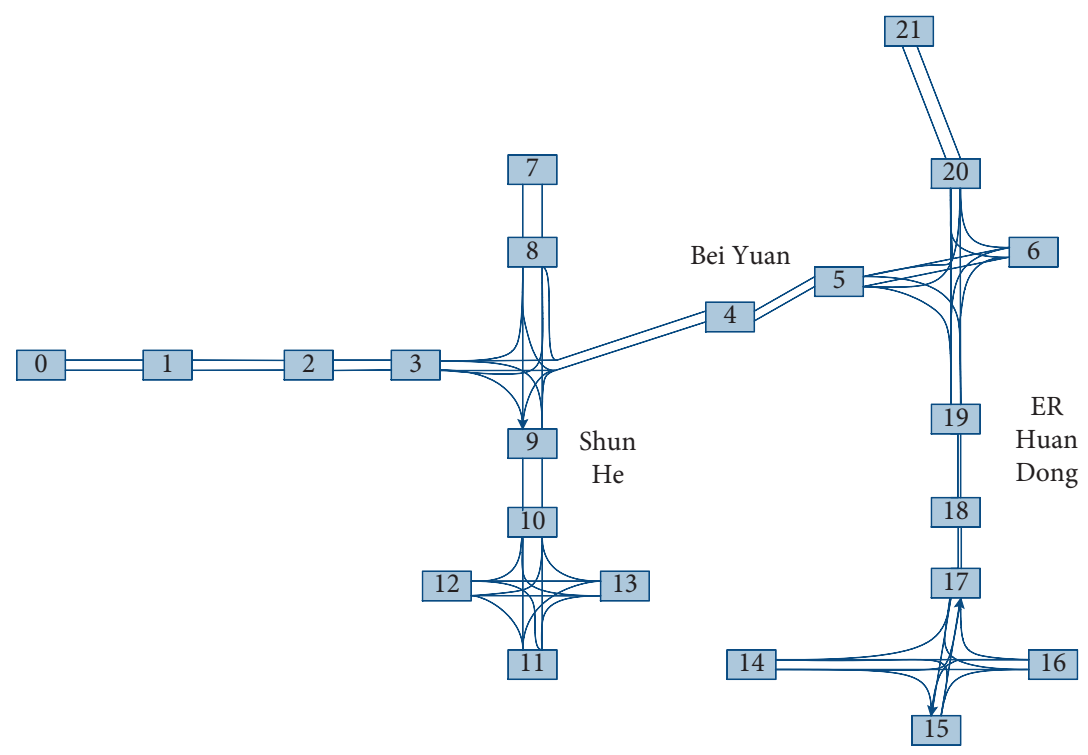

FiguRE 5: The elevated expressway network of Jinan.

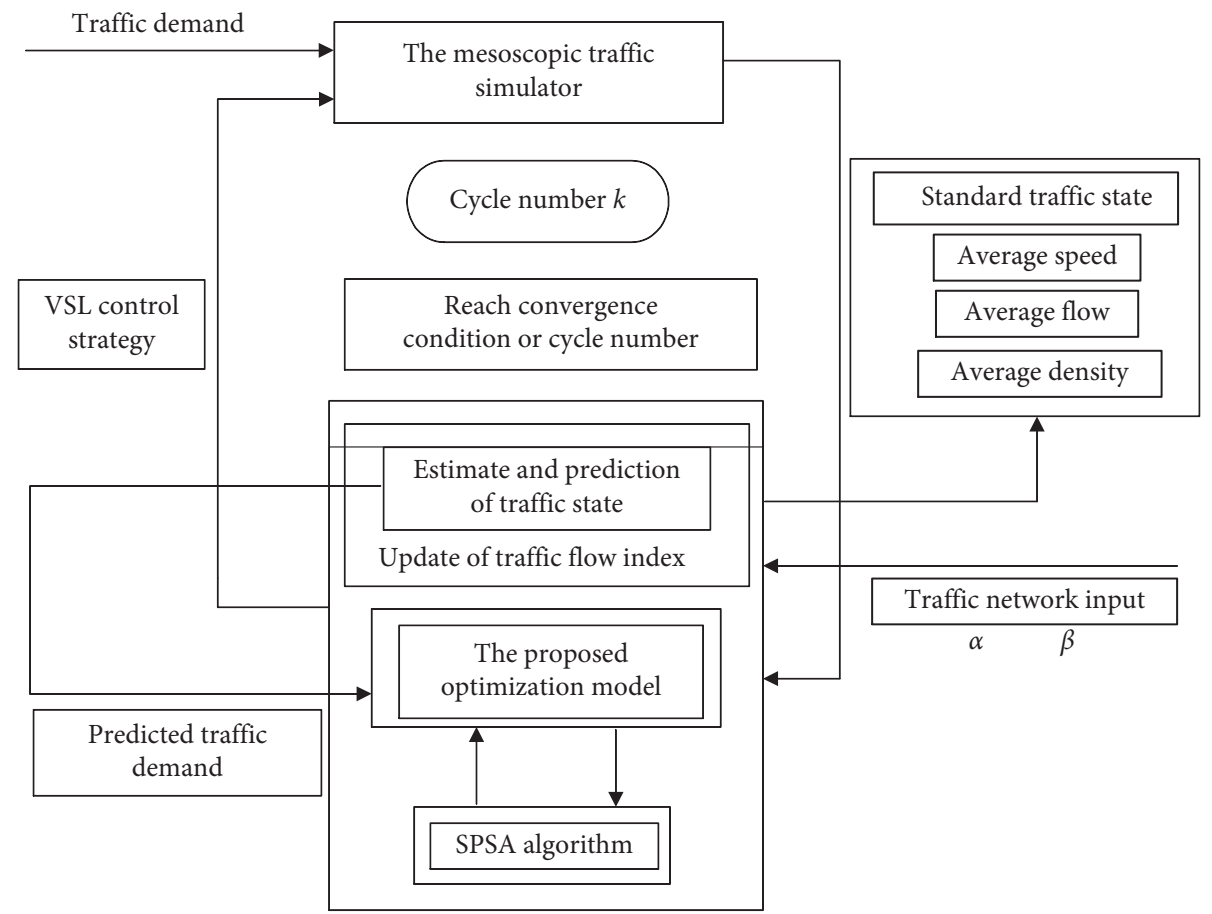

FIgURE 6: The simulation structure.

expressway connecting the network of the urban arteries, the speed of the traffic flow on the expressway should be decreased during the rush hour. Therefore, the initial value of variable speed limit is set on $7: 30$, from which the speed is limited to $60 \mathrm{~km} / \mathrm{h}$. From $8: 00$, the speed is limited to $50 \mathrm{~km} / \mathrm{h}$ and the speed is limited to $40 \mathrm{~km} / \mathrm{h}$ at $9: 00$. Then the above information variables have changed. The travelling time is $5092.6 \mathrm{~h}$; the total travelling distance is 157856.5 ; the total average speed is $31 \mathrm{~km} / \mathrm{h}$; the total traffic flow is 187008 per car unit; the total delay is $3194.3 \mathrm{~h}$; the total density is 6.3272 per car; the average traffic flow is 94.4721 cars. There is no traffic congestion under standard state. But when there is a control, the traffic congestion area reaches $19 \%$ of the whole network. According to the data, the traffic safety gets further ensured when there is a control over variable speed limit. However, the efficiency of the network operation decreases sharply. In particular, the traffic congestion occurs in some areas under network state. The whole network state improves greatly. From the perspective of microscopic aspect, the distribution of the average density, the average speed, and the average flow of each segment during each simulation time interval is shown in Figures 10-12, which 


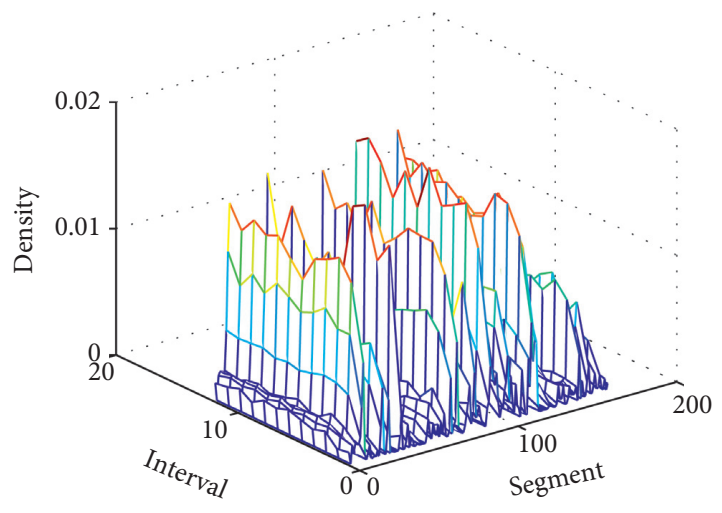

FIgURE 7: The averaged density without control.

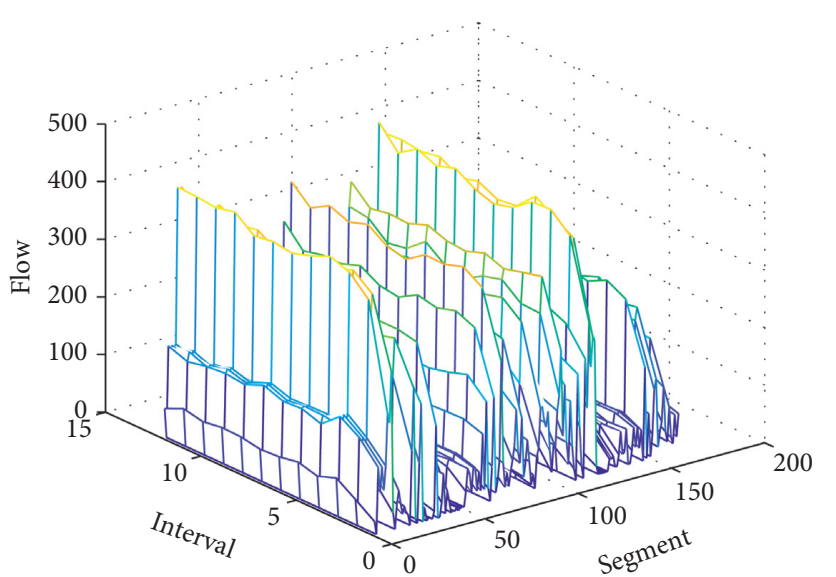

Figure 8: The averaged flow without control.

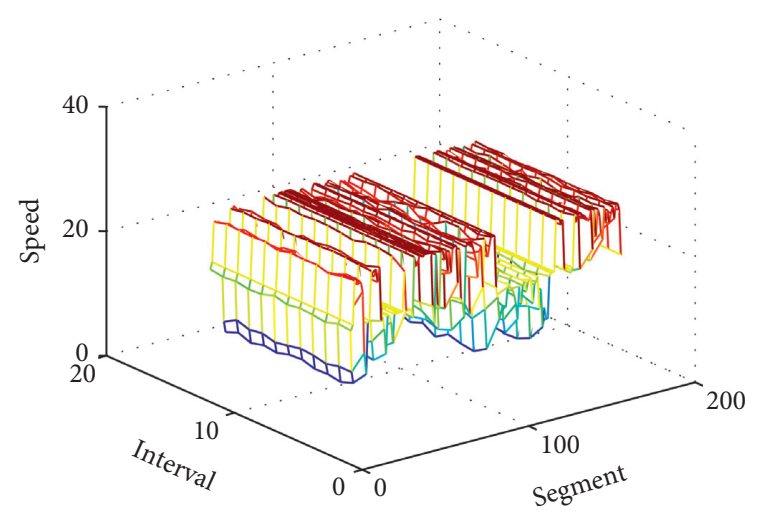

Figure 9: The averaged speed without control.

shows that the distribution of the network density is in imbalance and so do the distribution of speed and flow. Particularly, the average speed decreases dramatically. Thus, utilizing variable speed limit without control may lead to dramatic exacerbation of the traffic network. Obviously, the operation of the traffic flow can be adjusted through variable speed limit once there is a traffic accident. Therefore, the most satisfying scheme for setting control strategy of variable speed limit should be selected in reality.

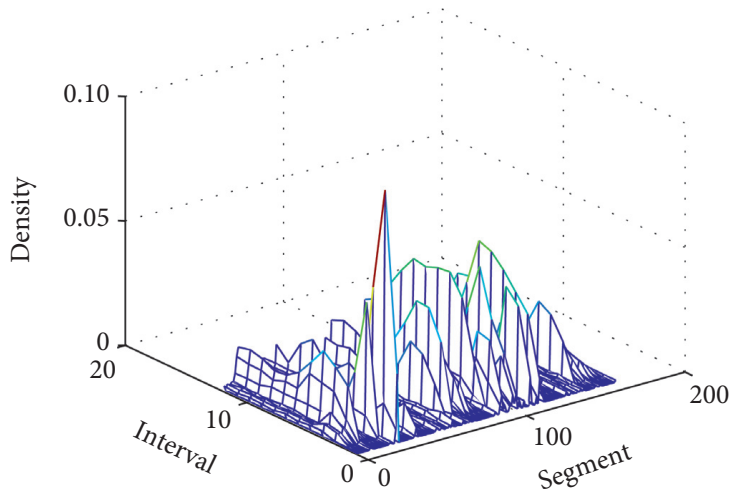

FIgURE 10: The averaged density with control.

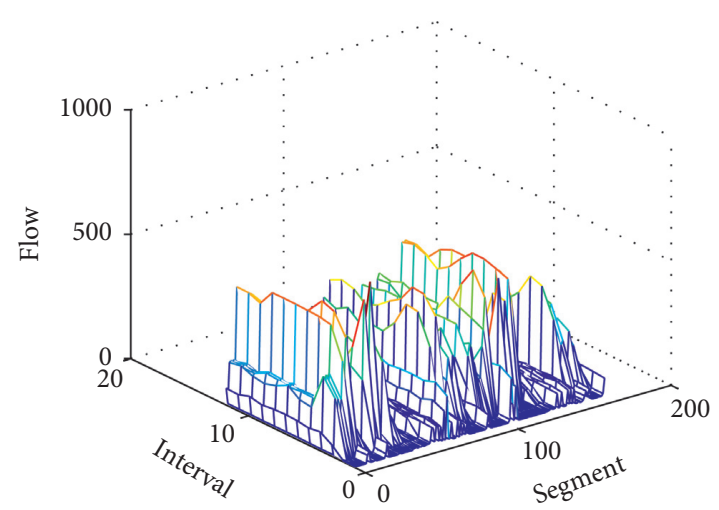

FIgURE 11: The averaged flow with control.

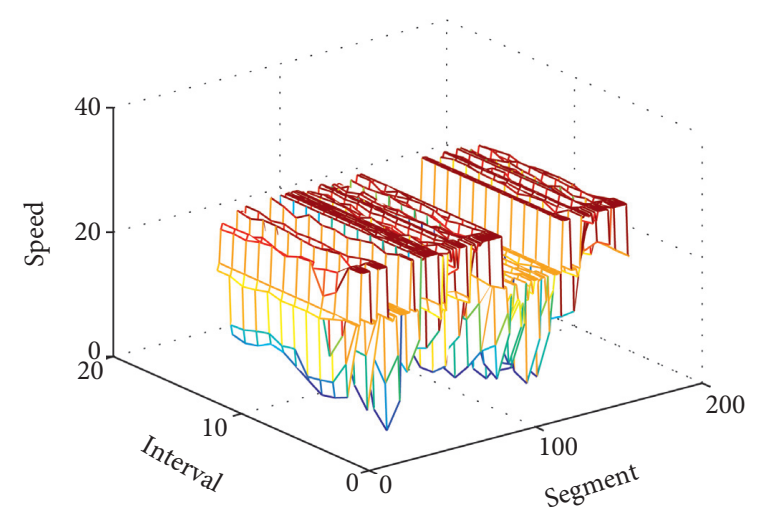

Figure 12: The averaged speed with control.

Under the above state, the implementation of the improved model of variable speed limit and the SPSA algorithm can figure out the most satisfying scheme of variable speed limit and then make it executed, thus testing all indexes of the traffic network. Again the above information has changed. The total travelling time is $3511.7 \mathrm{~h}$; the total travelling distance is $136520.4 \mathrm{~km}$; the total average speed is $36.8 \mathrm{~km} / \mathrm{h}$; the total traffic flow is 161809 cars; the total delay is $1870 \mathrm{~h}$; the largest delay at node is $337.2 \mathrm{~h}$; the total density 


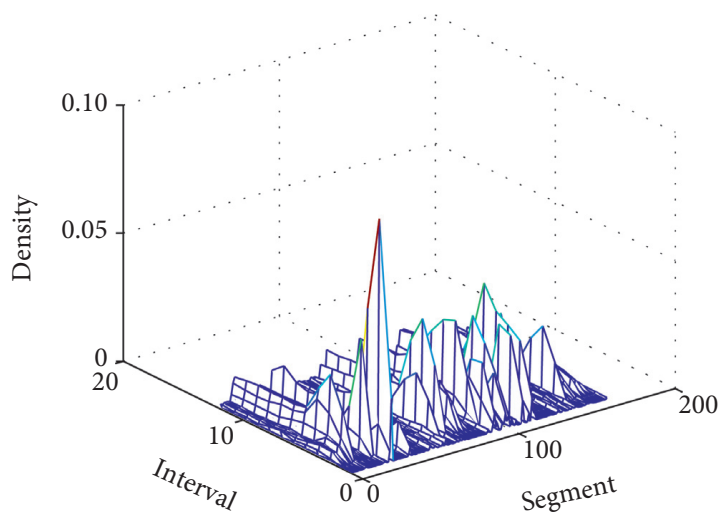

FIGURE 13: The optimal averaged density.

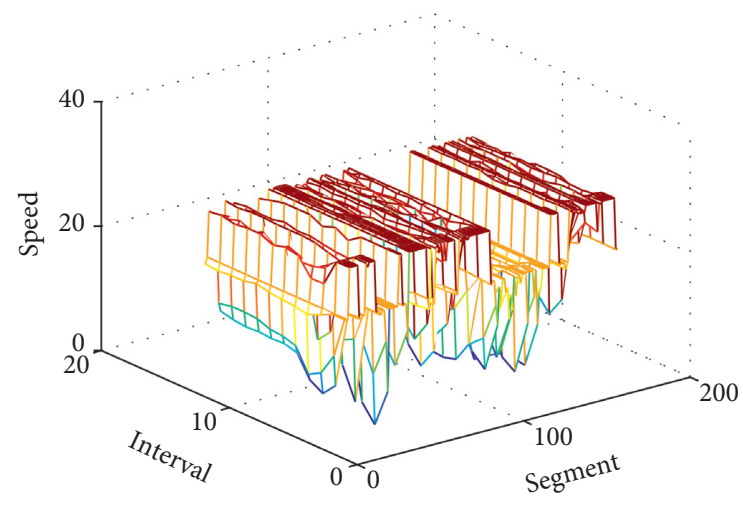

FIgURE 14: The optimal averaged speed.

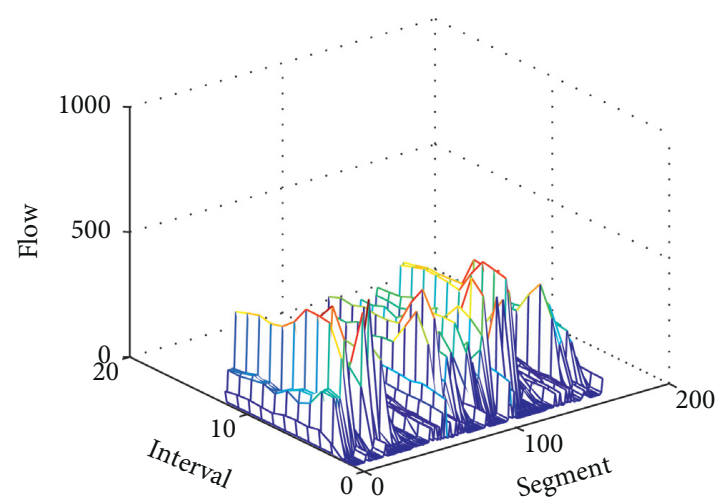

FIgURE 15: The optimal averaged flow.

is 4.7195 ; the average flow is 82.3237 cars. The congestion area is $1 \%$ of the total network under the state of optimized control. The light congestion road is $12 \%$. It can be seen that the density appears well-distributed in network compared with that of the control. The efficiency of vehicles' movement improves a lot. From the perspective of microscopic aspect, the distribution of the average density, the average speed, the average flow of each segment during each simulation time interval is shown in Figures 13-15. Apparently, the density, speed, and flow appear well-distributed and the average speed improves greatly. This saves passengers' time for travelling. Meanwhile, the VSL ensures the traffic safety by optimize the traffic network indexes and enables the traffic flow to move orderly.

The simulation results show that the VSL exerts considerable influence on the traffic flow distribution and the dynamic characteristics. The existence of the speed limitation in different time will inevitably lead to the decrease of the average speed. And with the increase of the total density, the total mileage of vehicles decreased, but it can be seen that the delay on the largest node does not increase significantly. If the proposed optimization control strategy is employed, it will decrease mostly the negative effect resulting from the speed limit and optimize the whole network of this area. According to the relevant study, the proper reduction of speed is beneficial for ensuring traffic safety and reducing the traffic accident.

It is worth noting that the movement of vehicle is in accordance with some regulations whether the model and algorithm are used in simulation test. This is similar to the fact that vehicles obey traffic rules and move orderly at intersections where there is no signal control. In reality, intersections with heavy traffic flow but no signal control, however, are usually the places where severe traffic congestion occurs. This often leads to vicious circle which results from violation against traffic rules and competition between drivers. Therefore, it must implement the control over traffic flow. However, the effect exerted by obeying traffic rules is much more admirable than that by control. Therefore, under the most satisfying state presented in this paper, the network state is much better than that of the time when there is no optimization but control implementation. Moreover, the efficiency of travelling improves rapidly. Compared to state without control, the network is much worse.

Although the time interval divided is a little longer, it does not affect the analysis of questions. The time interval can be shortened according to reality. This means that there is one more variable value domain in the SPSA algorithm, in which the advantage of this algorithm lies. The more the variables are, the more advantageous the algorithm is. That is to say, the speed and astringency of the algorithm have nothing to do with a variable number. The refinement of time interval is supportive to obtaining a better control strategy, therefore, making the control strategy over variable speed limit in each time interval more effective.

One of the objectives to employ VSL control is to maintain the traffic safety. The adjustment of speed according to time interval can contribute to not only traffic safety but also the travelling efficiency of road network. Secondly, the VSL can manage effectively the distribution of the traffic flow that always changes and guide the traffic flow for the sake of traffic structure. Thirdly, in some congestion areas, especially the places in which the travelling capacity of lanes decrease sharply (construction area; accident area), the VSL can be used to create a free flow area in the upstream, thus making traffic capacity of it equivalent to or slightly less than that of the largest lane group of this area. This can minimize the traffic congestion and the resulting chain reaction. 


\section{Research Conclusions and Prospects}

As an effective way to control the continuous traffic flow of freeway or expressway, the VSL control strategy can maintain traffic safety and adjust the distribution of the traffic flow. Considering the real time and accuracy of the VSL control strategy, this paper selects the mesoscopic traffic flow model as the study means, embeds VSL control strategy into the model, builds the improved mesoscopic traffic flow simulation model, and then designs its simulation flow. Next, the improved model of VSL control and the SPSAbased algorithm are proposed. The advantage of this algorithm lies in the fact that it can not only guarantee the accuracy of simulation but also meet online demands for real-time application. The simulation results show that the improved model and algorithm can enhance dramatically the efficiency of the network, the average speed, and the traffic capacity and enable the traffic density to appear welldistributed. Meanwhile, it can also ensure the traffic safety and be applied to such traffic circumstances as construction area or accident area. Compared to all vehicles that are intelligently equipped and obey unified traffic rules, the traffic network without any implementation of control strategy works better, which makes no sense in reality because areas without control strategy tend to be places in which the traffic congestion frequently occurs. The emphasis of the latter study is on the combination of VSL control strategy of urban expressway and signal control of urban arteries and initiation of aggregative model and algorithm. This is supportive for traffic sector to make decision.

\section{Data Availability}

The main data of this study are obtained by mesoscopic simulation software.

\section{Conflicts of Interest}

The authors declare that there are no conflicts of interest regarding the publication of this paper.

\section{Acknowledgments}

This work was supported by the National Natural Science Foundation of China (Grant nos. 71871130, 71771019, and 71971125); the University Science and Technology Program Funding Projects of Shandong Province (Grant no. J17KA211); the Social Science Foundation of Shandong Province (Grant no. 12BJJJ13); and the Science and Technology Program Funding Projects of Shandong Police College (Grant no. YSKYB2005).

\section{References}

[1] J. Mccrea and S. Moutari, "A hybrid macroscopic-based model for traffic flow in road networks," European Journal of Operational Research, vol. 207, no. 2, pp. 676-684, 2010.

[2] Y. Diao, Z. C. Juan, and A. N. Ni, "Review of mesoscopic traffic flow modeling and system simulation," Application Research of Computers, vol. 26, no. 7, pp. 2411-2415, 2009.
[3] S. B. Li, Research on the Run State Evaluation of Urban Transportation System and Control Strategies, Beijing Jiao Tong University, Beijing, China, 2012.

[4] D. F. Xie, Analyzing of Typical Problems of Urban Road Traffic Flow Based on Microscopic Methods, Beijing Jiao Tong University, Beijing, China, 2010.

[5] T. Wang, Research on Traffic Flow Modeling and Simulating Based on the Lattice Hydrodynamic Model, Beijing Jiao Tong University, Beijing, China, 2015.

[6] J. F. Tian, Z. Z. Yuan, J. Bin et al., "Phase transitions and the Korteweg-devries equation in the density difference lattice hydrodynamic model of traffic flow," International Journal of Modern Physics C, vol. 24, no. 3, Article ID 1350016, 2013.

[7] T. Wang, Z. Gao, W. Zhang, J. Zhang, and S. Li, "Phase transitions in the two-lane density difference lattice hydrodynamic model of traffic flow," Nonlinear Dynamics, vol. 77, no. 3, pp. 635-642, 2014.

[8] T. Wang, Z. Y. Gao, J. Zhang et al., "A new lattice hydrodynamic model for two-lane traffic with the consideration of density difference effect," Nonlinear Dynamics, vol. 75, no. 12, pp. 27-34, 2014.

[9] D. S. Felipe, V. Omer, and J. Auld, "Mesoscopic traffic flow model for agent-based simulation," Procedia Computer Science, vol. 151, pp. 858-863, 2019.

[10] J. Anahita, P. Ioannis, P. Markos et al., "A mesoscopic integrated urban traffic flow-emission model," Transportation Research Part C, vol. 75, pp. 45-83, 2017.

[11] A. A. Mohamed, R. J. Cunningham, V. V. Gayah et al., "Dynamic variable speed limit strategies for real-time crash risk reduction on freeways," Transportation Research Record, vol. 2078, no. 1, pp. 108-116, 2008.

[12] Y. Pu, L. Hu, Y. S. Jiang et al., "Variable speed-limit control before expressway mainline toll station," Journal of Traffic and Transportation Engineering, vol. 12, no. 5, pp. 119-126, 2012.

[13] J. Soobin, P. Chongmyung, and S. Dongmahn, "The multistation based variable speed limit model for realization on urban highway,” Electronics, vol. 9, no. 5, 2020.

[14] Y. Zhou, B. Lin, X. Yang et al., "Application of the bayesian model averaging in analyzing freeway traffic incident clearance time for emergency management," Journal of Advanced Transportation, vol. 2021, no. 4, 9 pages, Article ID 6671983, 2021.

[15] J. J. Zhang, M. B. Pang, and S. S. Ren, "Characteristic analysis of traffic flow in variable speed limit section of freeway based on cellular automaton model," Acta Physica Sinica, vol. 61, no. 24, pp. 340-347, 2012.

[16] W. Wang, Z. S. Yang, and D. X. Zhao, "Control model of variable speed limit based on finite horizon Markov Decisionmaking," Journal of Traffic and Transportation Engineering, vol. 11, no. 5, pp. 109-114, 2011.

[17] W. Y. Zhou, M. F. Yang, M. H. Lee et al., "Q-Learning-Based coordinated variable speed limit and hard shoulder running control strategy to reduce travel time at freeway corridor," Transportation Research Record, vol. 2674, no. 11, 2020.

[18] Y. Wang, X. Ma, Z. Li, Y. Liu, M. Xu, and Y. Wang, "Profit distribution in collaborative multiple centers vehicle routing problem," Journal of Cleaner Production, vol. 144, pp. 203219, 2017.

[19] Y. Wang, S. G. Peng, X. S. Zhou et al., "Green logistics location-routing problem with eco-packages," Transportation Research Part E: Logistics and Transportation Review, vol. 143, Article ID 102118, 2020.

[20] Y. Wang, Y. Y. Yuan, X. Guan et al., "Collaborative twoechelon multicenter vehicle routing optimization based on 
state-space-time network representation," Journal of Cleaner Production, vol. 258, Article ID 120590, 2020.

[21] J. C. Spall, "Multivariate stochastic approximation using a simultaneous perturbation gradient approximation," IEEE Transactions on Automatic Control, vol. 37, no. 3, pp. 332-341, 2002.

[22] J. C. Spall, "Implementation of the simultaneous perturbation algorithm for stochastic optimization," IEEE Transactions on Aerospace and Electronic Systems, vol. 34, no. 3, pp. 817-823, 1998. 